\title{
Assessment of Adherence to HIV Protease Inhibitors: Comparison and Combination of Various Methods, Including MEMS (Electronic Monitoring), Patient and Nurse Report, and Therapeutic Drug Monitoring
}

\author{
*Patricia W. H . Hugen, †Nienke Langebeek, *David M Burger, $\ddagger$ Bert Zomer, $†$ Rob van Leusen, \\ $\S$ Rob Schuurman, $\ddagger$ Peter P. Koopmans, and *Yechiel A. Hekster \\ *Departments of Clinical Pharmacy and $\ddagger$ General Internal Medicine, University Medical Centre Nijmegen, Nijmegen; \\ †Department of Internal Medicine, Rijnstate Hospital, Arnhem; and §Department of Virology, University Hospital Utrecht, \\ Utrecht, The Netherlands
}

Background: Adherence to protease inhibitor-containing antiretroviral therapy is crucial, but difficult to measure.

Objective: To compare and combine various methods of measuring adherence to the strict protease inhibitor-containing regimens.

Methods: The following methods were used: medication event monitoring system (MEMS) caps (electronic monitoring), therapeutic drug monitoring, pill count, pharmacy refill data, questionnaires, diaries (for registration of food patterns and special events related to the use of MEMS), adherence assessment by the physician and clinical nurse specialist, and in-depth interviews. In addition, ultrasensitive viral load and resistance testing was performed.

Results: Twenty-eight patients were included; data could be evaluated in 26. According to MEMS data, $25 \%$ of the patients took fewer than $95 \%$ of all doses, and two thirds of the patients took fewer than $95 \%$ of the doses on time. Only $43 \%$ of the patients showed good adherence with food restrictions. Methods that showed significant correlations with MEMS results were patients' self-reported adherence; therapeutic drug monitoring, indicating plasma levels outside predefined ranges; and estimation of adherence by a clinical nurse specialist, especially by in-depth interview.

Conclusion: Diary-corrected MEMS data gave a detailed insight into patients' adherence patterns. Patients' self-report and therapeutic drug monitoring were significantly correlated with the MEMS data, and the clinical nurse specialist may also play a role in identifying patients who are imperfectly adherent.

Key Words: Adherence-Electronic monitoring-HIV protease inhibitorsNurse-Plasma concentrations.
Although protease inhibitor-containing antiretroviral therapy has dramatically improved the outcomes for HIV-infected patients, suboptimal adherence to these complex regimens decreases the likelihood of suppress-

Address correspondence and reprint requests to Patricia W.H. Hugen, University Medical Centre Nijmegen, 533, Department of Clinical Pharmacy, PO Box 9101, 6500 HB Nijmegen, The Netherlands; e-mail: p.hugen@klinfarm.azn.nl

Manuscript received August 30, 2001; accepted February 20, 2002.

Financial support for this study was given by Abbott, GlaxoWellcome, Merck Sharp \& Dohme, and Roche. ing viral replication and increases the likelihood of developing resistance (1-15). High adherence seems necessary $(1,8,12,14)$, which means ingestion of the correct number of pills at the right time according to the prescribed food requirements. Adherence is further complicated by adverse events and factors such as stigmatization (16-19).

Whereas the virologic implications of suboptimal adherence are becoming increasingly clear, it is not yet apparent how adherence to these stringent regimens can best be measured. One problem in studying adherence is 
the lack of a gold standard (20-22). Thus far, self-report has predominantly been used $(5,6,8,10,13,23-27)$. Recently, a few studies using electronic monitoring have been presented (1,28-30). The medication event monitoring system (MEMS) records the opening and closing of a medication vial and thereby provides more detailed information. Nevertheless, it is still an indirect method because it does not measure drug ingestion. A direct, objective measure of ingestion of a drug is its plasma concentration $(20,31)$, although it only gives short-time information. Adherence with food requirements can be measured with a diary or other kinds of self-report.

The objective of our study was to combine several adherence-measuring methods to compare the information provided by these various methods. We aimed to find a set of methods that enables adherence monitoring of all relevant aspects of protease inhibitor-containing antiretroviral therapy. With the availability of tools to reliably monitor adherence, an important step toward developing effective interventions that improve adherence can be made.

\section{MATERIALS AND METHODS}

\section{Patients}

Two groups of outpatients were included in this study: 1) patients who were starting antiretroviral combination therapy including one or more protease inhibitors (naives) and 2) patients who were already using protease inhibitor-containing combination therapy for more than 48 weeks and who had an undetectable viral load (i.e., $<400$ or 500 copies/mL at the previous two measurements before start of the study; non-naives). In this descriptive study, the patient sample is a convenience sample, i.e., patient numbers were based on the availability of suitable patients in the two centers that participated, the University Medical Centre, Nijmegen and the Rijnstate Hospital, Arnhem. The goal was to include 20 naive and 20 non-naive patients, equally distributed over the two centers.

Patients had to be between 18 and 65 years of age and able to read and speak Dutch. During follow-up evaluation, patients continued their original medication regimen, although changes according to standard of care were allowed. Patients were monitored for 24 weeks during which three (for non-naives) or four (for naives) visits were planned, analogous to regular visits. Sociodemographic and other patient characteristics were registered during the first of these visits. If patients did not want to participate, the reasons for refusal, according to the patient and the nurse, were marked.

All participants signed written informed consent, and the study was approved by the local Ethics Committees of both study centers.

\section{Medication Event Monitoring System}

In this study, the MEMS was used, more specifically the eDEMs Track caps (Aardex, Zug, Switzerland), in combination with $200-\mathrm{mL}$ pill bottles. Medication dosing times were retrieved from the cap and analyzed by the Powerview program (Aardex, Zug, Switzerland).

For our study, we used an intermediate version of Powerview that enabled us to insert and delete events to correct the MEMS data. These corrections were necessary because the bottle was also opened during study visits and for refills, although generally no medication was taken at those times. In these cases, data were corrected based on notes from the diary or on the study visit forms.

Patients who used a medication cassette to organize their protease inhibitor ingestions and who were not willing to stop this practice during the study were excluded, although patients were allowed to take their medication out of the MEMS bottle before the actual ingestion time, if only sporadically. MEMS data were also corrected for these occurrences.

The MEMS caps were supplied only for the protease inhibitors. During the study, regular prescriptions were used. Medication was supplied by the patient's local pharmacy and registered according to the regular method at that particular pharmacy. Patients performed the refills themselves. They were explicitly instructed only to use the MEMS bottle and to make notes of deviations. Also, they were told to ingest the medication directly after taking it from the bottle. Patients were aware of the function of the MEMS bottle.

Parameters extracted from the Powerview report were percentage of prescribed medication taken, percentage of medication taken on schedule (i.e., within 1 hour before or after the scheduled time), and percentage of days on which the correct number of doses was taken. The calendar plot (overview of number of doses taken each day) and chronology plot (schematic report of dose-timing) were also visually inspected.

\section{Questionnaires}

Questionnaires were completed by patients at every planned study visit, i.e., at baseline and at 12- and 24-week follow-up evaluations. For naive patients, an extra questionnaire was supplied at week 4 , and an adjusted questionnaire was given at baseline. The questions were completed without supervision of the physician or nurse.

The names of antiretroviral agents used, with dosing regimen and food restrictions, were noted on the questionnaire. In addition, patients assessed their adherence based on ingestion of the correct number of doses and pills, the timing of ingestion, and adherence with food restrictions (visual analog scale from 1 [not precise] to 10 [very precise]).

After completion, the questionnaires were placed in a sealed envelope and sent directly to the investigator. The mean estimate over the various visits was calculated.

\section{Diaries}

Patients were given six 31-day diaries. With respect to measuring adherence, the diary contained the following items: timing and composition of meals (scale $1-5$ for amount and fat content, examples were given in the instructions) and specific issues related to MEMS use (participants had to mark refills, device use, and other unscheduled openings). The use of diaries in this study was not intended for measuring adherence regarding ingestion. Patients were asked to update their diaries four times a day.

Analysis of adherence with food requirements was done by relating what the patient reported to consider as food requirements in the questionnaire to the data concerning food in the diary and the MEMS ingestion times. We looked at general food patterns over the entire study period and performed a detailed analysis of five randomly chosen days per patient. The interval between medication and food intake and the composition with respect to portion and fat content were compared with the requirements (indinavir, minor or no food intake 2 hours before and 1 hour after ingestion of medication; other regimens, intake of medication within 2 hours of food intake). Thus, patients were 
categorized into three groups: (1) good (no deviation), (2) moderate (minor deviations, irregularly), and (3) poor (frequent or major deviations) compliance with food restrictions.

\section{Adherence Assessment by Treating Physician and Clinical Nurse Specialist}

At each visit, the treating physician and clinical nurse specialist assessed the adherence of the patient in the same way the patient had done in the questionnaire (based on ingestion of correct number of doses and pills, timing of ingestion, and adherence with food restrictions; visual analog scale from 1 [not precise] to 10 [very precise]). The mean estimate over the various visits was calculated.

\section{Plasma Drug Concentrations}

Plasma samples were drawn at each visit, including unplanned visits, to determine protease inhibitor concentrations. Two weeks after start of the follow-up period, an 8-hour pharmacokinetic profile was recorded. The samples were analyzed with a validated high-performance liquid chromatography (HPLC) method, with a lower limit of quantitation of $.04 \mathrm{mg} / \mathrm{L}$ (32). Random plasma concentrations were compared with the expected concentration at the corresponding time after ingestion, i.e., the interval between dose intake and plasma sampling according to the patient. For a comparison with the individual curve, deviation from the expected concentrations was calculated by determining the median absolute deviation from the expected ratio $1(1=$ equal to concentration after observed ingestion). For a comparison with population curves, predefined limits, known as concentration ratio limits (CORALS), were used. CORALS generally reflect plasma concentrations 2-3 times higher than reference population values after observed ingestion or lower than one third to one sixth of these population values. Concentrations outside these limits have been found to be predictive for nonadherence (33). For each patient, the percentage of samples outside these limits was determined.

\section{Pill Count and Pharmacy Refill Data}

At each visit, patients were asked to bring their antiretroviral medication to the hospital for a pill count. The number of pills in the MEMS bottle and those in the unopened and opened bottles were counted and registered separately.

On the second part of the pill count form, the prescriptions given at that particular visit were registered. By combining pill count and prescription data, the percentage of doses taken of the protease inhibitor could be calculated.

At the end of follow-up period, the pharmacy refill data for each patient were requested at the local pharmacy. Most pharmacy refill reports already include a calculation of the date on which the stock will be finished. From this refill report, the regularity of the refill pattern could be deduced. Pill count and refill data were combined to calculate the percentage of doses taken. The pill count at baseline reflected the initial stock; the one at the end of follow-up period, the final stock. The in-between refills reported by the local pharmacy reflected new stock. The following formula was used to calculate the percentage taken.

(initial stock +

$\begin{aligned} & \text { refilled amount) }- \text { final stock } \\ & \text { number of pills per day } * \\ & \text { number of days in follow-up }\end{aligned} \times 100=\%$ of pills taken

\section{In-Depth Interview}

An in-depth interview by the clinical nurse specialist was held with a subgroup of patients at the end of follow-up period. Patients had to give consent for the interview at the start of the study; patients who either refused or stopped prematurely were replaced.

The interview was semi-structured: a list of questions was supplied to give direction to the conversation. The interviews were recorded on tape, and the patients were told that no one besides the investigators would hear the interview and that the interview would be processed anonymously.

After transposition, statements regarding the patients' adherence were extracted by two researchers. By combining these statements, patients' adherence was categorized as 1) good (no deviations in ingestion, timing, or food requirements reported), 2) moderate (minor deviations reported), or 3) poor (patient admitting skipping doses, irregular drug intake, or deviations from food requirements).

\section{Viral Load and Resistance Analysis}

In addition to standard viral load measurements (cut off, 400 or 500 copies $/ \mathrm{mL}$ ), ultrasensitive measurements (cut-off, 25 copies $/ \mathrm{mL}$; Ultrasensitive HIV-1 Cobas Amplicor Monitor Assay, Roche Diagnostics, Pleasantin, CA, U.S.A.) were performed at baseline and at the end of follow-up period. If the viral load at the end of follow-up period was $>1000$ copies $/ \mathrm{mL}$, genotypic resistance analysis was performed using an automated sequencer (ABI 377, PE Biosystems, Foster City, CA, U.S.A.) and Big-Dye-terminator chemistry (PE Biosystems). Population sequencing of the entire protease gene and of the RT gene from amino acid 1-300 was performed using polymerase chain reaction (PCR) amplified genome fragments derived from plasma virus RNA (34).

\section{Statistics}

Statistics were performed with SPSS for Windows (v. 9.0, SPSS Inc., Chicago, IL, U.S.A.). To compare the various methods with MEMS data, nonparametric Spearman correlation coefficients (r) were calculated with their corresponding levels of significance. Spearman $r$ values of $<.35$ were regarded insufficient, whereas $r$ values of $0.6-0.7$ were high enough to consider the method comparable with MEMS data. $p<$ .05 was regarded as significant. To compare a combination of methods with MEMS, categoric data of the specific methods were multiplied and then Spearman correlation coefficients were calculated again. Categorization of the data was done to increase the sensitivity of the methods to discriminate poor adherence from moderate or good adherence, although some methods did not result in numerical data. The categories were based on the literature or extracted from the data. The following cut-off points were used: MEMS $\%$ taken and $\%$ of days correct number of doses taken, 1 (good adherence): $\geq 95 \%, 2$ (moderate) : $95 \%-90 \%, 3$ (poor): $<90 \%$; MEMS $\%$ on schedule, $>90 \%, 90 \%-$ $80 \%$, and $<80 \%$; self-reported adherence/physician and nurse estimate, $>9.0,9.0-8.0, \leq 8.0$; pill count/refill data, $1: \geq 95 \%, 2: 95 \%-90 \%, 3$ : $<90 \%$; plasma concentrations, 1: $0 \%$ plasma concentrations outside CORALS, $3:>0 \%$; in-depth interviews, categorized based on the statements of the patients concerning their own adherence.

\section{RESULTS}

\section{Patients}

Twenty-eight patients were included in the study: 21 non-naive, 7 naive. Non-naive patients had a median 
duration of antiretroviral therapy use of 2.5 years (interquartile range [IQR], 2.0-3.3). Thirteen patients used a twice-daily regimen; the other 15 used a thrice-daily dosing frequency. Indinavir taken alone and the combination of ritonavir + saquinavir were the most frequently used protease inhibitors (12 and 10 patients, respectively). All patients but 1 were male, and all but 1 white; most (19 of 28) were homosexual.

Twenty-six patients completed follow-up week 12, and 24 completed follow-up week 24 . Four of the 28 patients stopped prematurely (before follow-up week 24) because of protease inhibitor toxicity (1 naive, 2 nonnaive patients) or patient request (1 non-naive patient). Two of these patients stopped therapy before follow-up week 4 and were not included in the analyses.

\section{Medication Event Monitoring System}

According to the corrected MEMS reports (corrected for notes in diary, during visits, etc.), the median percentage of prescribed doses taken (\% taken) was $98.9 \%$ (range, $10.1 \%-102.0 \%$ ); the percentage of days on which the correct number of doses was taken (\% correct days) was $94.6 \%$ (range, $4.1 \%-99.5 \%$ ), and the percentage of doses taken on schedule (\% on schedule, within 1 hour before or after the scheduled time) was $91.1 \%$ (range, $6.4 \%-100.3 \%$ ). Twenty-five percent of the patients took fewer than $95 \%$ of all doses; $50 \%$ of the patients took the correct number of doses fewer than $95 \%$ of the days, and two thirds of the patients took fewer than $95 \%$ of the doses on time (Fig. 1). Figure 2 shows an example of perfect and imperfect compliance as depicted in MEMS chronology plots.

\section{Questionnaires}

From the questionnaire, the patient's dosing regimen was extracted and used to analyze the MEMS data. In 4 cases $(15 \%)$, food restrictions, according to the patients, were different than advised. Median patients' selfreported adherence (average of several measurements per patient) was 8.6 on a scale from 1 to 10 (IQR, 8.0 9.3). The lowest mark given was 5.7.

\section{Diaries}

Of the 26 patients, 1 patient did not take enough doses to analyze his accompanying food pattern, 1 did not sufficiently complete the food part of the diary, and 3 patients reported not to be restricted to food requirements. Of the other 21 patients, $9(43 \%)$ complied well with the food restrictions, 4 (19\%) complied moderately, and 8

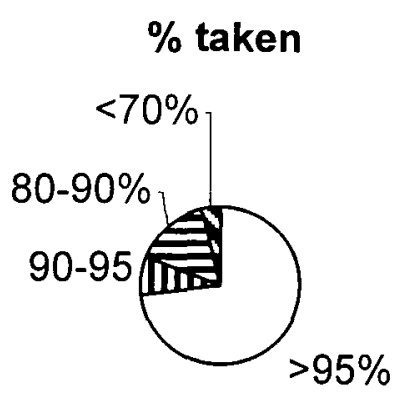

\section{$\%$ days with correct $\mathrm{nr}$ of doses}

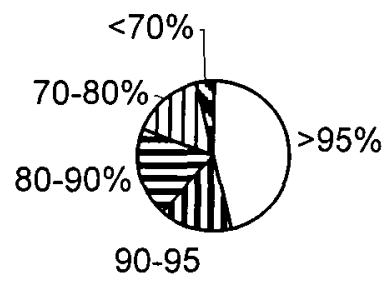

\section{$\%$ on time}

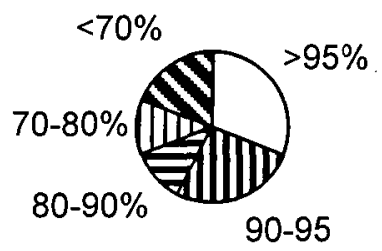

FIG. 1. Distribution of adherence over the study population. Twenty-seven percent of the patients took fewer than $95 \%$ of all doses; $54 \%$ took the correct number of doses for fewer than $95 \%$ of the days, and $69 \%$ took fewer than $95 \%$ of the doses on time (within 1 hour before or after the scheduled time).

(38\%) poorly. Of the evaluative patients, 11 had to take their medication on (nearly) empty stomach, whereas 10 patients had to take their medication with (fat) food. Adherence with food restrictions in the first group was comparable with that in the second group $(p=.495$, Mann-Whitney $U$ test).

\section{Adherence Assessment by Treating Physician and Clinical Nurse Specialist}

The median adherence as reported by the nurse was 8.8 (IQR, 7.9-9.3) and by the physician 9.0 (IQR, 8.3- 


\section{Chronology}

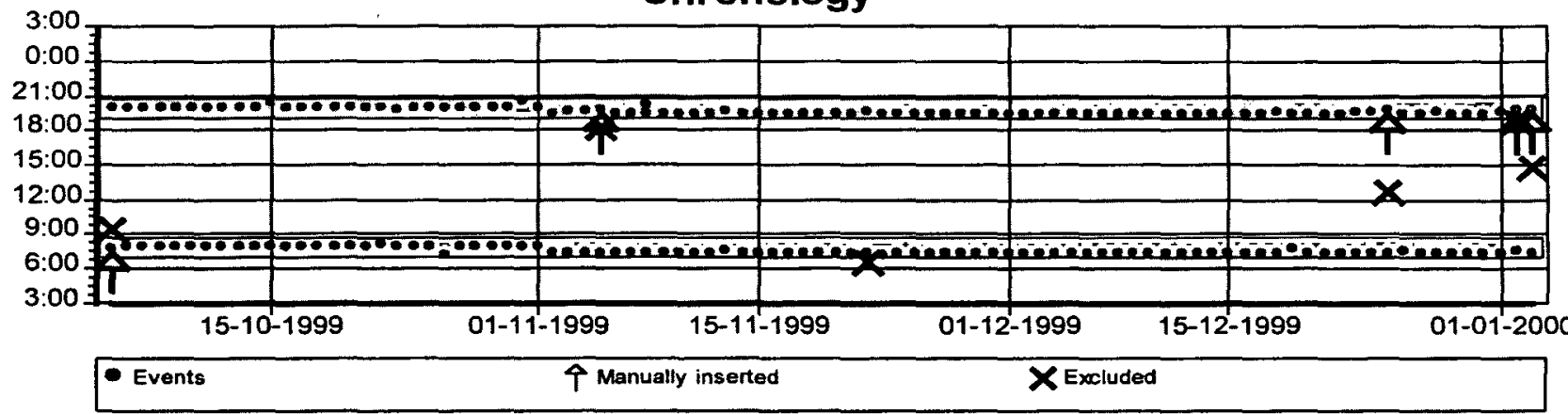

\section{Missing doses}

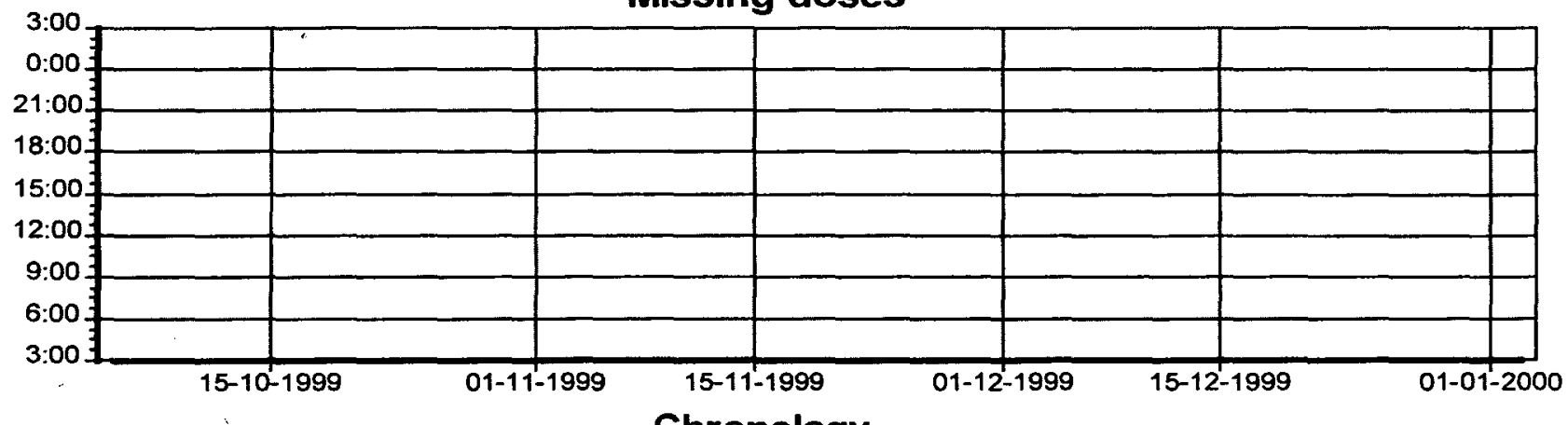

\section{Chronology}
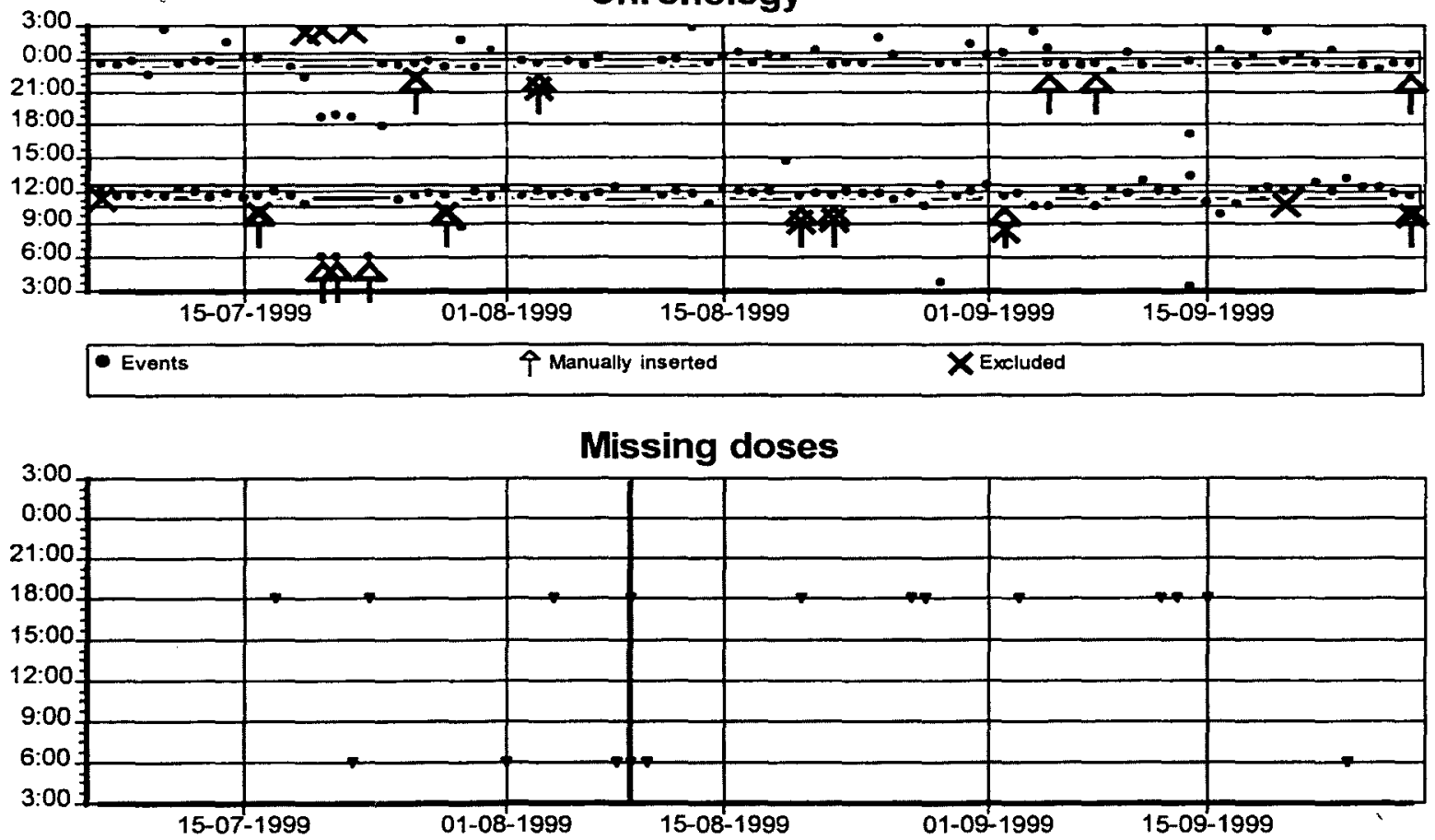

FIG. 2. Chronology plot and missing doses of 2 patients on a twice-daily schedule. The upper two panels are from a patient with perfect adherence (taken, 101\%; on schedule, $100 \%$; days with correct number of doses, $99 \%$ ). The lower two panels are from a patient with imperfect adherence (taken, $92 \%$; on schedule, $77 \%$; days with correct number of doses, $81 \%$ ). Time is depicted on the $Y$ axis; the date, on the $X$ axis. Manually inserted and excluded events are based on notes in the diaries and visit forms. The grey horizontal bars represent the permitted time interval of the scheduled doses $( \pm 1$ hour $)$. 
9.6). The nurses did not give anyone a score below 6.0 compared with the 7.1 low score given by the physicians.

\section{Plasma Drug Concentrations}

The mean number of plasma samples per patient was $5.5(\mathrm{SD}, 1.9)$. The number of evaluative samples was 143 .

Deviations in protease inhibitor concentrations compared with intrapatient reference values recorded under supervised ingestion ranged from $10 \%$ to $167 \%$, with a median deviation of $36 \%$. Using CORALS, the percentage of abnormal plasma concentrations among patients ranged from $0 \%$ to $88 \%$ (median, $0 \%$ ), with 6 patients (23\%) having one or more abnormal plasma concentrations. Two patients $(8 \%)$ had one or more plasma concentrations below the lower limit of quantitation.

\section{Pill Count and Pharmacy Refill Data}

When pill count and pharmacy refill data were combined, the median percentage of pills taken was $100 \%$ (range, $71 \%-132 \%$ ). Thirty percent of the patients were regarded as overadherent. In a small pilot study of 5 patients, a detailed analysis of the pill count and pharmacy refill data was performed. Several problems with these methods were encountered. First, there were strong indications that the pill count data had been manipulated, as has also been found by others, by the patient with the poorest compliance according to the MEMS data (35). For example, MEMS openings were recorded shortly before visits, although no medication had been ingested at that time as was shown by undetectable plasma levels. Also, prescriptions were refilled shortly before visits, although the pill count forms indicated that this patient had had insufficient stock much earlier. Most likely the stock that the patient was assumed to have at home was brought to the hospital, and the excess was left at home. Nevertheless, it was difficult to determine the patient's actual behavior from the pill count and refill data, and the zombination provides an enormous overestimation of the adherence according to MEMS (101 [pill count/refill] vs. 10\% [MEMS] taken).

Problems encountered with other patients from the piot study were mixing of stocks among partners who ssed the same medication, inaccurate reporting of prescriptions by nurses, patients having old prescriptions at lome, patients forgetting to bring the medication to the 1ospital, either purposely or accidentally.

\section{In-Depth Interviews}

The clinical nurse specialist held an in-depth interview with 14 of the 26 patients. One patient (7\%) was catego- rized as having poor compliance; $6(43 \%)$ were categorized as having moderate compliance, and the other 7 $(50 \%)$ as having good compliance.

\section{Correlation Between Different Methods}

Table 1 shows all individual results as numeric values, if available, and categorized as well, as described in the Methods section. Table 2 lists the Spearman correlation coefficients $(\rho)$ of the MEMS data compared with the other methods.

According to the plasma concentration data obtained from the CORALS listed in the tables, protease inhibitor plasma concentrations deviated more from intrapatient reference values, recorded under supervised ingestion, in patients who were less adherent according to the MEMS data (\% taken: Spearman $\rho,-.426 ; p<.05 ; \%$ on time: Spearman $\rho,-.527 ; p<.01)$. In addition to assessing adherence in itself, plasma concentrations added relevant information to MEMS in cases where the MEMS data reported ingestion but plasma levels were undetectable or vice versa, and they were an objective check for the accuracy of the MEMS data.

After adherence with food had been categorized as (1) good (no violation of food restrictions), (2) moderate (minor violations), or (3) poor (repetitive and more serious violations), the correlation with the categorized MEMS data was as follows: MEMS \% taken: Spearman $\rho=.559, p<.01 ;$ MEMS $\%$ on time: Spearman $\rho=$ $.673, p<.01$.

Methods that showed relatively high and significant correlations with the MEMS results were patients' selfreported estimate of adherence in the anonymous questionnaire $(\rho, .73 ; p<.001)$, plasma levels outside predefined ranges $(\rho, .77 ; p<.001)$, and, to a lesser extent, estimation of adherence by the clinical nurse specialist $(\rho, .57 ; p<.01)$. A combination of these methods did not increase the relation with the MEMS results $(\rho, .57 ; p<$ .01 , to $\rho, .72 ; p<.001)$. The physician's estimate of adherence correlated less well $(\rho, .43 ; p<.05)$, and the combination of pill count and pharmacy refill data only correlated significantly with the MEMS data when overadherence was judged as poor adherence (categorized on the basis of the absolute deviation from 100\%; $\rho, .43 ; p<.05$ ). In a subgroup, in-depth interviews with the clinical nurse specialist resulted in a high correlation with the MEMS results $(\rho, .78 ; p<.01)$.

\section{Acceptance of Study Methods}

The following comments on the study methods were made: the MEMS vial was too large to handle (200-mL 
TABLE 1. Comparison of adherence measured by various methods (adherence category numbers in parentheses)

\begin{tabular}{|c|c|c|c|c|c|c|c|c|c|c|c|c|c|}
\hline Patient & $\begin{array}{l}\text { Protease } \\
\text { inhibitor }\end{array}$ & BID/TID & $\begin{array}{l}\text { Non- } \\
\text { naive/ } \\
\text { naive }\end{array}$ & $\begin{array}{l}\text { MEMS, } \\
\text { \% taken }\end{array}$ & $\begin{array}{l}\text { MEMS, } \\
\% \text { days }\end{array}$ & $\begin{array}{l}\text { MEMS, } \\
\% \text { on time }\end{array}$ & $\begin{array}{l}\text { Food } \\
\text { (diary) }\end{array}$ & $\begin{array}{l}\text { Questionnaire } \\
\text { (self-report) }\end{array}$ & $\begin{array}{l}\text { Physician } \\
\text { estimate }\end{array}$ & $\begin{array}{l}\text { HIV nurse } \\
\text { estimate }\end{array}$ & $\begin{array}{c}\text { Pill count } \\
+ \text { refills }(\%)\end{array}$ & $\begin{array}{l}\text { Plasma } \\
\text { level }^{e}\end{array}$ & Interview \\
\hline $1^{a}$ & IDV & 3 & $\mathrm{NN}$ & $97(1)$ & $87(3)$ & $80(2)$ & 2 & $9.0(2)$ & $10.0(1)$ & $9.0(2)$ & $\mathrm{ND}^{a}$ & $0(1)$ & ND \\
\hline 2 & IDV & 3 & $\mathrm{NN}$ & $100(1)$ & $99(1)$ & $92(1)$ & 1 & $7.0(3)$ & $9.0(2)$ & $8.6(2)$ & $100(1)$ & $0(1)$ & ND \\
\hline 3 & $\mathrm{NFV}+\mathrm{SQV}$ & 2 & $\mathrm{NN}$ & $86(3)$ & $74(3)$ & $78(3)$ & 3 & $8.0(3)$ & $8.7(2)$ & $8.3(2)$ & $86(3)$ & $50(3)$ & ND \\
\hline $4^{a . c}$ & NFV & 2 & $\mathrm{NN}$ & $94(2)$ & $87(3)$ & $90(2)$ & - & $9.0(2)$ & $8.5(2)$ & $8.5(2)$ & $\mathrm{ND}^{\prime \prime}$ & $0(1)$ & ND \\
\hline 5 & $\mathrm{RTV}+\mathrm{SQV}$ & 2 & $\mathrm{NN}$ & $99(1)$ & $97(1)$ & $95(1)$ & 1 & $9.3(1)$ & $10.0(1)$ & $9.3(1)$ & $98(1)$ & $0(1)$ & ND \\
\hline 6 & IDV & 3 & $\mathrm{NN}$ & $98(1)$ & $94(2)$ & $93(1)$ & 1 & $8.3(2)$ & $10.0(1)$ & $9.3(1)$ & $10091)$ & $0(1)$ & l \\
\hline $7^{\circ}$ & $\mathrm{RTV}+\mathrm{SQV}$ & 2 & $\mathrm{NN}$ & $99(1)$ & $99(1)$ & $99(1)$ & - & $9.3(1)$ & $9.0(2)$ & $10.0(1)$ & $99(1)$ & 0 (1) & 1 \\
\hline $8^{r}$ & RTV + SQV & 2 & $\mathrm{NN}$ & $10(3)$ & $4(3)$ & $6(3)$ & - & $8.0(3)$ & $7.9(3)$ & $7.7(3)$ & $101(1)$ & $88(3)$ & ND \\
\hline 9 & $\mathrm{RTV}+\mathrm{SQV}$ & 2 & $\mathrm{NN}$ & $88(3)$ & $76(3)$ & $67(3)$ & 3 & $7.7(3)$ & $8.5(2)$ & $8.0(3)$ & $126(1)$ & $50(3)$ & 3 \\
\hline 10 & $\mathrm{RTV}+\mathrm{SQV}$ & 2 & $\mathrm{NN}$ & $100(1)$ & $95(1)$ & $93(1)$ & 3 & $9.0(2)$ & $8.3(2)$ & $8.2(2)$ & $100(1)$ & $25(3)$ & 1 \\
\hline 11 & IDV & 3 & $\mathrm{NN}$ & $99(1)$ & $88(3)$ & $83(2)$ & 1 & $8.0(3)$ & $8.3(2)$ & $8.8(2)$ & $71(3)$ & $0(1)$ & ND \\
\hline 12 & IDV & 3 & NN & $98(1)$ & $95(1)$ & $78(3)$ & 3 & $5.7(3)$ & $8.8(2)$ & $7.4(3)$ & $98(1)$ & $0(1)$ & 2 \\
\hline 13 & IDV & 3 & $\mathrm{NN}$ & $97(1)$ & $84(3)$ & $81(2)$ & 2 & $8.0(3)$ & $7.6(3)$ & $9.3(1)$ & $100(1)$ & $0(1)$ & 2 \\
\hline 14 & IDV & 3 & $\mathrm{NN}$ & $99(1)$ & $98(1)$ & $87(2)$ & 2 & $9.0(2)$ & $9.8(1)$ & $9.4(1)$ & $\mathrm{ND}^{\prime \prime}$ & $0(1)$ & 2 \\
\hline $15^{c}$ & $\mathrm{RTV}+\mathrm{SQV}$ & 2 & $\mathrm{NN}$ & $83(3)$ & $71(3)$ & $31(3)$ & - & $7.7(3)$ & $7.1(3)$ & $6.0(3)$ & $121(1)$ & $14(3)$ & $N D^{b}$ \\
\hline 16 & SQV & 3 & NN & $102(1)$ & $91(2)$ & $69(3)$ & 3 & $9.0(2)$ & $9.1(1)$ & $8.7(2)$ & $93(2)$ & $0(1)$ & ND \\
\hline 17 & $\mathrm{RTV}+\mathrm{SQV}$ & 2 & NN & $101(1)$ & $98(1)$ & $100(1)$ & 2 & $10.0(1)$ & $10.0(1)$ & $9.5(1)$ & $87(3)$ & $0(1)$ & 1 \\
\hline $18^{c}$ & $\mathrm{RTV}+\mathrm{SQV}$ & 2 & $N N$ & $99(1)$ & $97(1)$ & $98(1)$ & - & $10.0(1)$ & $9.2(1)$ & $9.3(1)$ & $101(1)$ & $0(1)$ & ND \\
\hline 19 & IDV & 3 & NN & $100(1)$ & $97(1)$ & $97(1)$ & 1 & $10.0(1)$ & $7.7(3)$ & 7.7 (3) & $101(1)$ & $0(1)$ & 1 \\
\hline \multirow[t]{2}{*}{20} & IDV & 3 & $\mathrm{NN}$ & $99(1)$ & $97(1)$ & $92(1)$ & 3 & $10.0(1)$ & $9.3(1)$ & $10.0(1)$ & $100(1)$ & $0(1)$ & 2 \\
\hline & & & & Stopped & & & & & & & & & \\
\hline 21 & RTV + IDV & 2 & $\mathrm{NN}$ & $<4$ weeks & $\mathrm{x}$ & $\mathrm{x}$ & $\mathrm{x}$ & $\mathrm{x}$ & $\mathrm{x}$ & $\mathrm{x}$ & $x$ & $\mathrm{x}$ & $x$ \\
\hline 22 & IDV & 3 & NA & $100(1)$ & $97(1)$ & $97(1)$ & 1 & $9.8(1)$ & $9.6(1)$ & $9.3(1)$ & $95(1)$ & $0(1)$ & 2 \\
\hline 23 & NFV & 2 & NA & $99(1)$ & $98(1)$ & $96(1)$ & 1 & $8.5(2)$ & $9.1(1)$ & $9.8(1)$ & $132(1)$ & $0(1)$ & 1 \\
\hline 24 & IDV & 3 & $\mathrm{NA}$ & $94(2)$ & $81(3)$ & $90(2)$ & 3 & $8.8(2)$ & $9.3(1)$ & $9.5(1)$ & $80(3)$ & $0(1)$ & 2 \\
\hline 25 & $\mathrm{RTV}+\mathrm{SQV}$ & 2 & $\mathrm{NA}$ & $100(1)$ & $100(1)$ & $93(1)$ & 1 & $8.8(2)$ & $8.2(2)$ & $7.5(3)$ & $126(1)$ & $0(1)$ & ND \\
\hline 26 & $\mathrm{RTV}+\mathrm{SQV}$ & 2 & NA & $99(1)$ & $98(1)$ & $97(1)$ & 1 & $9.0(2)$ & $9.6(1)$ & $8.8(2)$ & $100(1)$ & $0(1)$ & 1 \\
\hline \multirow[t]{2}{*}{27} & NFV & 2 & NA & $88(3)$ & $73(3)$ & $62(3)$ & 3 & $7.7(3)$ & $8.5(2)$ & $7.2(3)$ & $92(2)$ & $50(3)$ & $\mathrm{ND}^{b}$ \\
\hline & & & & Stopped & & & & & & & & & \\
\hline 28 & IDV & 3 & NA & $<4$ weeks & $x$ & $\mathrm{x}$ & $\mathrm{x}$ & $\mathrm{x}$ & $\mathrm{x}$ & $\mathrm{x}$ & $x$ & $x$ & $\mathrm{x}$ \\
\hline
\end{tabular}

MEMS \% taken: percentage of doses taken according to MEMS. MEMS \% days: percentage of days on which the correct number of doses was taken. MEMS \% or time: percentage of doses taken within 1 hour before or after the scheduled ingestion time.

Adherence categories: $1=$ good adherence; $2=$ moderate adherence; $3=$ poor adherence

a Patient 1 stopped medication between 12 and 24 weeks of follow-up observation; patient 4 withdrew consent at 12 weeks of follow-up observation.

b Patient 15 eventually refused cooperation, patient 27 was too depressed at the time of the interview.

"Patients 4, 7, and 18 reported to have no food restrictions, patient 8 had not enough ingestions to enable analysis of his food pattern, and the diary registration of patien 15 was too poor to enable analysis of his food pattern.

${ }^{d}$ No refill data available.

"Percentage of plasma concentration outside CORALS (concentration ratio limits).

IDV, indinavir; NFV, nelfinavir; RTR, ritonavir; SQV, saquinavir; NN, non-naive; NA, naive; ND, not done.

bottles were used because of the large number of pills); having only one vial was inconvenient, and not being able to use a medication cassette was problematic. Keeping the diary was problematic, especially adding entries four times a day. Specific items with respect to MEMS were scored well, but items that had to be scored more often, such as the amount and fat content of food, were more difficult for some patients. As a result, several patients filled in all items once daily. The pill count was accepted well, although in practice, patients sometimes did not bring their pills with them.

\section{Viral Load and Resistance Analysis}

For 23 of the 26 patients, ultrasensitive viral load data (cut off 25 copies $/ \mathrm{mL}$ ) were available at the end of follow-up period. In 2 patients, the viral load was detectable. One of these patients (non-naive, number 14 in the table) had 1290 copies $/ \mathrm{mL}$. According to MEMS, this patient took $99 \%$ of the doses, and $87 \%$ were taken or time. Deviations in timing were small but occurred regu. larly. However, the viral load increase may also be ex. plained by a relatively low indinavir exposure, which was seen even after observed ingestion. Resistancr analysis showed resistance to zidovudine and abacavir although zidovudine was only used before the study.

A second non-naive patient (number 8 in the table had 3635 copies $/ \mathrm{mL}$; this patient had the poorest adher ence ( $10 \%$ taken according to MEMS and probably ever less according to undetectable plasma concentrations af ter MEMS opening). This patient's virus was resistan for zidovudine, abacavir, and lamivudine but not for pro tease inhibitors. He had used zidovudine and lamivudin during the study and long before. The viral loads of th other 21 patients were undetectable $(<25$ copies $/ \mathrm{mL})$ although 5 patients ( 3 non-naive and 2 naive) had takes fewer than $95 \%$ of their doses (the lowest \% taken wa $83 \%$ ) and 8 (7 non-naive, 1 naive) had taken fewer thas 
TABLE 2. Correlations between MEMS results and other methods to measure adherence

\begin{tabular}{|c|c|c|c|c|}
\hline \multirow[b]{2}{*}{ Method } & \multicolumn{2}{|c|}{ MEMS (\% taken) } & \multicolumn{2}{|c|}{ MEMS (\% taken on time) } \\
\hline & $\begin{array}{l}\text { Spearman } \\
\text { rho numeric }\end{array}$ & Ordinal & $\begin{array}{l}\text { Spearman } \\
\text { rho numeric }\end{array}$ & ordinal \\
\hline Questionnaire (self-report) & $0.552^{c}$ & $0.538^{c}$ & $0.721^{d}$ & $0.731^{d}$ \\
\hline Plasma concentrations $>$ CORALS & $-0.568^{c}$ & $0.768^{d}$ & $-0.593^{c}$ & $0.543^{c}$ \\
\hline Nurse estimate & 0.287 & $0.472^{b}$ & $0.567^{c}$ & $0.530^{c}$ \\
\hline Physician estimate & 0.273 & $0.426^{b}$ & $0.429^{b}$ & 0.387 \\
\hline \multicolumn{5}{|l|}{ Pill count/refill data } \\
\hline Percentage & -0.074 & 0.284 & 0.027 & 0.382 \\
\hline Deviation from $100 \%$ & -0.146 & 0.414 & -0.229 & $0.430^{b}$ \\
\hline In-depth interview ${ }^{a}$ & $\mathrm{x}$ & $0.550^{*}$ & $\mathrm{x}$ & $0.778^{\circ}$ \\
\hline \multicolumn{5}{|l|}{ Combination of methods } \\
\hline $\mathrm{Q} \times \mathrm{PI} \times \mathrm{N}$ & $\mathrm{x}$ & $0.618^{c}$ & $\mathrm{x}$ & $0.684^{d}$ \\
\hline $\mathrm{Q} \times \mathrm{PI}$ & $\mathrm{x}$ & $0.642^{d}$ & $\mathrm{x}$ & $0.721^{d t}$ \\
\hline $\mathrm{Q} \times \mathrm{N}$ & $\mathrm{x}$ & $0.569^{c}$ & $x$ & $0.705^{d}$ \\
\hline $\mathrm{PI} \times \mathrm{N}$ & $\mathrm{x}$ & $0.610^{b}$ & $\mathrm{x}$ & $0.586^{d}$ \\
\hline
\end{tabular}

For the ordinal results the categorization as listed in Table 1 is used. Additionally, the pill count/refill data are calculated as the absolute deviation from $100 \%$ and categorized by dividing the deviations into 3 equal groups.

${ }^{a}$ In-depth interviews were held in 14 of the 26 patients. CORALS, concentration ratio limits; Q, questionnaire; PI, plasma concentrations; $\mathrm{N}$, nurse estimate. Combination of methods was calculated by multiplying the category numbers.

${ }^{b} p<.05$.

${ }^{c} p<.01$.

${ }^{d} p<.001$.

$90 \%$ of their doses on time $(31 \%-83 \%)$ according to MEMS.

\section{DISCUSSION}

In this study, diary-corrected MEMS data gave a detailed and accurate insight into adherence patterns of HIV-infected patients using protease inhibitorcontaining antiretroviral therapy. Patients' self-report and therapeutic drug monitoring were significantly and highly correlated with adherence according to MEMS, as was the case for the in-depth interviews held by the clinical nurse specialist.

\section{Medication Event Monitoring System}

The MEMS caps were only supplied for the protease inhibitors because this class of medication has the most stringent requirements with respect to dose frequency, timing, and food intake. Nucleoside reverse transcriptase inhibitors are less vulnerable, and although nonnucleoside analogs are at high risk for development of resistance, their long elimination half-lives make them less prone to nonadherence.

Because of the different appearance of the MEMS bottle and cap and the refill necessity at home, telling the patients about the function of the MEMS caps could not be avoided. For this reason, and others, a follow-up pe- riod of 24 weeks was chosen: we assumed it to be unlikely for patients to mimic perfect adherence for a long period $(36,37)$.

By using MEMS caps, various patterns of adherence were seen among different patients. Specific problems such as missing afternoon doses, variation in the timing of the bedtime dose, and changed timing during weekends were seen. Such detailed information will enable a targeted intervention to improve adherence (38).

\section{Self-Report and In-Depth Interview}

Self-report is often regarded as a method that overestimates adherence $(13,29,30)$. However, an estimate of poor adherence by a patient should be regarded seriously (21); several studies have demonstrated an association between self-report and viral outcome $(10,12,23,29)$.

When patients were asked anonymously about their adherence in our study, their estimate corresponded relatively well with the results from the MEMS caps. Particularly, patients who repeatedly gave themselves an 8 or lower on a visual analog scale from 1 to 10 appeared to be imperfectly adherent, which proves that it is useful to ask patients about their adherence. This request may be done in writing by a third party, i.e., not by the treating physician, but, for example, by a pharmacist.

The physician too often gave a wrong estimate of the patient's adherence to turn his or her judgment into a 
useful method, as has been shown before $(1,28)$. The nurse was better able to judge the patient's adherence. When the nurse held an in-depth interview with the patient and remarks with respect to adherence were extracted to categorize the patients' adherence, a rather strong correlation with adherence according to MEMS was found. This finding could mean that the approachability of and the close relationship with the nurse, as mentioned by patients in their interviews, reveals detailed and reliable information on adherence.

\section{Therapeutic Drug Monitoring}

When looking at plasma concentrations drawn after unobserved ingestion, the patients with larger deviations from their individual pharmacokinetic profile after observed ingestion appeared to be less adherent according to MEMS. Additionally, we saw a strong relation between having plasma concentrations outside predefined population concentration limits assessed after observed ingestion (i.e., CORALS) (33) and nonadherence, especially with respect to the number of doses taken. Five of the 6 patients with one or more plasma concentrations outside the CORALS took less than $90 \%$ of their medication according to MEMS, and thus were detected by repeated therapeutic drug monitoring. These 5 patients were the only ones who were categorized as having poor compliance (MEMS, <90\% taken, category 3 ).

A disadvantage of plasma sampling is that patients could mimic perfect adherence by taking doses shortly before a visit $(37,39)$. Although this still may cause abnormal plasma concentrations, for example, because no autoinduction has occurred during the days of nonadherence, dose-timing is wrong, or extra doses are taken to compensate for missed doses (40), there is a chance that nonadherence will not be detected. Nevertheless, the results of the current study show that when patients knew that their plasma concentrations were determined, deviating plasma concentrations were found that correlated with imperfect adherence according to MEMS. This result agrees with the observation that the MEMS reports did not show an improvement in adherence shortly before study visits. The absence of this "toothbrush effect" has been described before (36).

\section{Pharmacy Refill Data and Pill Count}

Only a rough estimation of the refill pattern could be extracted from the pharmacy refill data (3). Most patients in this study were in the non-naive group and may have had a stock of pills at home. The number of pills in this stock is unknown if no pill count is performed, and thus the exact situation cannot be assessed with refill data alone (35). Only a combination of pill count and pharmacy refill data could give an estimation of adherence in this study. Nevertheless, even by combining pill count and pharmacy refill data, a percentage of adherence could be calculated that may appear rather good, but that, when reviewing the stocks at several time points, shows overuse or underuse, indicating that the overall calculation is actually inaccurate. This finding agrees with the poor correlation between adherence according to MEMS and adherence according to pill count and refill data in the current study and with the limitations of the methods recognized by others $(3,4,20,31,40,41)$.

\section{Diaries}

Analysis of adherence with food restrictions registered in the diaries was based on the food requirements reported by the patient in the questionniaire because instructions may have been incorrect. From the analysis of food patterns in the diaries, it became clear that violation of the requirements occurred for the drugs that had to be taken with food (fat) and those that had to be taken on a nearly empty stomach.

It was found that keeping a diary, especially when it has to be completed several times per day, is problematic for patients and therefore cannot be applied to larger patient groups. Monitoring adherence with food restrictions is difficult to perform. One approach is to have the clinical nurse specialist discuss this issue with the patient.

\section{Adherence Rate}

Our patient group may seem highly adherent compared with the adherence rates found by others $(1,3,13$, 29,36 ), but we suspect that $11 \%-16 \%$ of the patients who refused to participate would have been nonadherent; in other words, we did not select an extremely adherent patient group. A more likely explanation for the relatively high adherence may be found in the inclusion criteria: only patients without communication problems were included, and in the majority of the participants, their therapy thus far had been effective-one of the causes of good efficacy may be good adherence.

\section{Viral Load}

From the viral load data in this study, it is noted that virologic failure occurred only in the patient with the poorest compliance during the 24-week study period. Other patients reached or kept undetectable viral loads, 
even though adherence was sometimes lower than the threshold necessary for virologic suppression as suggested by others $(1,8,12,14)$. However, the viral load results from this study are only indicative because the study group is heterogeneous, with respect to duration of therapy, kind of pretreatment, and pretreatment viral load. The latter was not always known and may be important for the grade of adherence needed to reach and maintain viral suppression. Nevertheless, patients with lower adherence are at risk for virologic failure and possible development of virologic resistance (1-14), although no thresholds can be determined from this study, and exact relations are not yet fully understood.

In conclusion, the MEMS data give a detailed insight into patients' adherence patterns. Notes about special events occurring in relation to MEMS caps, such as visit openings, refills at home, or device use, should be used to correct the MEMS data to improve their accuracy (42). Therapeutic drug monitoring can be used as a direct measure to objectify the MEMS results, whereas plasma concentrations outside predefined limits are highly correlated with the MEMS results and thus can be used to detect nonadherent patients. Another method that showed a highly significant correlation with the MEMS results was the patients' self-reported estimate of adherence in an anonymous questionnaire and, to a lesser extent, the estimation of adherence by a clinical nurse specialist. In a random subgroup, in-depth interviews with the clinical nurse specialist resulted in a high correlation with the MEMS results, which could mean that by allowing patients talk to a person they trust and who is easily accessible may help to reveal nonadherence. Thus, therapeutic drug monitoring, patients' self-report, and clinical nurse specialist assessment of adherence after an in-depth interview with the patient can be used to detect patients who have a problem with adherence, and MEMS can be used in problematic patient groups in whom interventions are planned because MEMS give a real insight into adherence patterns. In addition to MEMS, diaries should be used, plasma concentrations should be measured, and patients should always be asked about their adherence, preferably by a clinical nurse specialist or a third party, such as a pharmacist.

Acknowledgments: The authors thank clinical nurse specialists Arnold Coors, Yvonne Vincken, and Karin Grintjes for their help in realizing this study. The authors also thank the HIV internists of the UMC Nijmegen and Rijnstate Hospital Arnhem for their adherence judgments, and the technicians of the Department of Clinical Pharmacy for measuring the plasma drug concentrations. The authors thank Aardex for providing the intermediate Powerview version. Additionally, the authors thank Roche Diagnostics for the supply of materials for the ultrasensitive viral load test, and clinical psychologist Jan Ver- coulen for his advice on the questionnaires and diaries. The authors also thank Marijcke Schoots van de Siepkamp for transposition of the in-depth interviews.

\section{REFERENCES}

1. Paterson DL, Swindells S, Mohr J, et al. Adherence to protease inhibitor therapy and outcomes in patients with HIV infection. Ann Intern Med 2000;133:21-30.

2. Vanhove GF, Schapiro JM, Winters MA, et al. Patient compliance and drug failure in protease inhibitor monotherapy. JAMA 1996 ; 276:1955-6.

3. Maher K, Klimas $\mathbf{N}$, Fletcher MA, et al. Disease progression, adherence, and protease inhibitor therapy for HIV infection in an Urban Veterans Affairs Medical Center. J Acquir Immune Defic Syndr 1999;22:358-63.

4. Grimes RM, Easling I, Ray $S$, et al. Non-adherence to protease inhibitors in 758 patients from 3 separate cohorts of HIV infected patients in Houston, Texas [abstract ThPeB4983]. 13th International Aids Conference, Durban, South Africa, July 9-14, 2000.

5. Wutoh AK, Brown CM, Jones T, et al. Associations between treatment behaviors and viral load among older HIV-infected adults [abstract ThPeB4971]. 13th International Aids Conference, Durban, South Africa, July 9-14, 2000.

6. Nieuwkerk PT, Gisolf EH, Danner SA, et al. Impact of patient adherence on viral suppression in a randomized clinical trial [abstract 441]. 7th European Conference on Clinical Aspects and Treatment of HIV-Infection, Lisbon, Portugal, October 23-27, 1999.

7. Cato A, Cavanaugh J, Shi H, Hsu A, Leonard J, et al. The effect of multiple doses of ritonavir on the pharmacokinetics of rifabutin. Clin Pharmacol Ther 1997;63:414-21.

8. Balfour L, Khaliq Y, Gayton J, et al. Adherence to HIV protease inhibitor therapy and maximal suppression of plasma viremia: when is adherence enough? Aids Impact 1999, Ottawa, Canada, July $15-17,1999$.

9. Bangsberg D, Hecht FM, Charlebois E, et al. Adherence to protease inhibitors, HIV-1 viral load, and development of drug resistance in an indigent population. AIDS 2000;14:357-66.

10. Le Moing V, Masquelier B, Moatti JP, et al. To study predictors of immunologic response to PI therapy, along with virologic response including adherence to therapy [abstract 596]. 39th ICAAC, San Francisco, California, September 26-29, 1999.

11. Deeks $\mathrm{S}$, Loftus $\mathrm{R}$, Cohen $\mathrm{P}$, et al. Incidence and predictors of virologic failure to indinavir or/and ritonavir in an urban health clinic [abstract LB-2]. 37th ICAAC, Toronto, Ontario, Canada, September 28-October 1, 1997.

12. Haubrich RH, Little SJ, Currier JS, et al. The value of patientreported adherence to antiretroviral therapy in predicting virologic and immunologic response. AIDS 1999;13:1099-107.

13. Golin C, Liu H, Hays R, et al. Self-reported adherence to protease inhibitors substantially overestimates an objective measure [abstract 95]. 6th Conference on Retroviruses and Opportunistic Infections, Chicago, Illinois, January 31-February 4, 1999.

14. Kaplan A, Golin C, Beck K, et al. Adherence to protease inhibitor therapy and viral load [abstract 096]. 6th Conference on Retroviruses and Opportunistic Infections, Chicago, Illinois, January 31-February 4, 1999.

15. Tuldra A, Fumaz CR, Ferrer MJ, et al. Predicting long-term adherence to HAART [abstract 512]. 7th European Conference on Clinical Aspects and Treatment of HIV-Infection, Lisbon, Portugal, October 23-27, 1999.

16. Weidle PJ, Ganea CE, McGowan JP, et al. Multiple reasons for nonadherence to antiretroviral medications: need for a multifaceted approach to improve adherence [abstract 32360]. 12th World Aids Conference, Geneva, Switzerland, June 28-July 3, 1998.

17. Stewart KE, Call SA, Cloud GA, et al. Prospective prediction of antiretroviral adherence: the role of patient-perceived barriers [ab- 
stract 587]. 39th ICAAC, San Francisco, California, September 26-29, 1999.

18. Knobel H, Rubio R, Miro JM, et al. Adherence to antiretroviral therapy: the patient's perspective [abstract 857]. 7th European Conference on Clinical Aspects and Treatment of HIV-Infection, Lisbon, Portugal, October 23-27, 1999.

19. Quirk J, Wilks J. Patient compliance on combination HIV antiretroviral therapies [abstract 32384]. 12th World Aids Conference, Geneva, Switzerland, June 28-July 3, 1998.

20. Grymonpre RE, Didur CD, Montgomery PR, et al. Pill count, self-report, and pharmacy claims data to measure medication adherence in the elderly. Ann Pharmacother 1998;32:749-54.

21 . US Health \& Human Services. Adherence to potent antiretroviral therapy [online]. Available at: http://www.medscape.com/govmt/ dhhs/guidelines/hiv/hiva. Accessed October 18, 1999.

22. Wagner GJ, Rabkin JG. Measuring medication adherence: is assessment of missed doses sufficient? AIDS 1999;13:2491-2.

23. Demasi R, Tolson J, Pham S, et al. Self-reported adherence to HAART and correlation with HIV RNA: initial results with the patient medication adherence questionnaire [poster 94]. 6th Conference on Retroviruses and Opportunistic Infections, Chicago, Illinois, January 31-February 4, 1999.

24. Gordillo V, Amo dJ, Soriano V, et al. Sociodemographic and psychologic variables influencing adherence to antiretroviral therapy. AIDS 1999;13:1763-9.

25. Luber AD, Mulnick J, Raber S, et al. Patient adherence with twice daily dosing of nelfinavir equivalent to non-nucleoside reverse transcriptase inhibitors when given in combination with dual nucleoside analogues [abstract ThPeB4991]. 13th International Aids Conference, Durban, South Africa, July 9-14, 2000.

26. Murri R, Ammassari A, Gallicano $K$, et al. Patient-reported nonadherence to HAART is related to protease inhibitor levels. $J A c$ quir Immune Defic Syndr 2000;24:123-8.

27. Eldred LJ, Wu AW, Chaisson RE, et al. Adherence to antiretroviral therapy and Pneumocystis prophylaxis in HIV disease. $J$ Acquir Immune Defic Syndr Hum Retrovirol 1998;18:117-25.

28. Miller L, Liu H, Beck K, et al. Providers' estimates of adherence overestimate reports from medication event monitoring system (MEMS) for patients on protease inhibitors [poster 97]. 6th Conference on Retroviruses and Opportunistic Infections, Chicago, Illinois, January 31-February 4, 1999.

29. Bangsberg DR, Hecht FM, Charlebois EC, et al. Spontaneous ad- herence audits predict viral suppression in the REACH cohort [abstract 93]. 6th Conference on Retroviruses and Opportunistic Infections, Chicago, Illinois, January 31-February 4, 1999.

30. Melbourne KM, Geletko SM, Brown SL, et al. Medication adherence in patients with HIV infection: a comparison of two measurement methods. AIDS Reader 1999;9:329-38.

31. Stockwell Morris L, Schulz RM. Patient compliance-an overview. $J$ Clin Pharmacol Ther 1992;17:283-95.

32. Hugen PWH, Verwey-van Wissen CPWGM, Burger DM, et al. Simultaneous determination of the HIV-protease inhibitors indinavir, nelfinavir, saquinavir and ritonavir in human plasma by reversed-phase high-performance liquid chromatography. J Chromatogr B Biomed Sci Appl 1999;727:139-49.

33. Hugen PWH, Burger DM, Aarnoutse RE, et al. Concentration ratios of protease inhibitors can be applied to assess noncompliance [abstract 3.1]. First International Workshop on Clinical Pharmacology of HIV Therapy, Noordwijk, The Netherlands, March 30-31, 2000.

34. Nijhuis M, Boucher CAB, Schuurman R. A sensitive one-tube RT-PCR for the amplification of HIV RNA. Biotechniques 1994; 19:323-7.

35. Gregoire J-P, Guilbert R, Archambault A, et al. Measurement of non-compliance to antihypertensive medication using pill counts and pharmacy records. J Soc Admin Pharm 1997;14:198-207.

36. Kastrissios H, Suarez JR, Katzenstein D, et al. Characterizing patterns of drug-taking behavior with a multiple drug regimen in an AIDS clinical trial. AIDS 1998;12:2295-2303.

37. Urquhart J. The electronic medication event monitor: lessons for pharmacotherapy. Clin Pharmacokinet 1997;32:345-56.

38. Matsuyama JR, Mason BJ, Jue SG. Pharmacists' interventions using an electronic medication-event monitoring device's adherence data versus pill counts. Ann Pharmacother 1993;27:851-5.

39. Cramer JA, Scheyer RD, Mattson RH. Compliance declines between clinic visits. Arch Intern Med 1990;150:1509-10.

40. Rudd P, Ahmed S, Zachary V, et al. Improved compliance measures: applications in an ambulatory hypertensive drug trial. Clin Pharmacol Ther 1990;48:676-85.

41. Cramer JA, Mattson RH, Prevey ML, et al. How often is medication taken as prescribed? JAMA 1989;261:3273-7.

42. Arnet I, Haefeli WE. Overconsumption detected by electronic drug monitoring requires subtle interpretation. Clin Pharmacol Ther 2000;67:44-7. 